\title{
Three-dimensional MHD modeling of waves in active region loops
}

\author{
Leon Ofman $\dagger$ and Małgorzata Selwa \\ Catholic University of America, \\ NASA Goddard Space Flight Center, Code 671 \\ Washington, DC 20064, USA
}

\begin{abstract}
Observations show that MHD waves are one of the most important universal processes in the heliosphere. These waves are likely to play an important role in energy transfer in the heliosphere, and they can be used as a diagnostic tool of the properties of the local magneto-fluid environment. Recent observations by TRACE and Hinode satellites provide ample evidence of oscillations in coronal active region loops. The oscillations were interpreted as fast (kink), slow, and Alfvén modes, and the properties of the waves were used for coronal seismology. However, due to the complex interactions of the various modes in the inhomogeneous active region plasma, and due to nonlinearity, idealized linear theory is inadequate to properly describe the waves. To overcome this theoretical shortcoming we developed 3D MHD models of waves in active region loops. We investigated the effects of 3D active region magnetic and density structure on the oscillations and the wave dissipation, and we investigated the oscillation of individual loops. Some loops were constructed to contain several threads and twist. Here, we present the results of our models, and show how they can be used to understand better the properties of the waves, and of the active regions.
\end{abstract}

Keywords. Sun: activity, Sun: corona, Sun: flares, Sun: magnetic fields, MHD, waves

\section{Introduction}

MHD waves were recently observed in detail in coronal loops (Aschwanden et al. 1999; Nakariakov et al. 1999; Verwichte et al. 2004; Ofman \& Wang 2008), and throughout the heliosphere. These waves have been studied for decades as a possible source of energy for coronal heating and solar wind acceleration. The properties of the observed waves and the geometry of the loops can be used for coronal seismology, i.e., the determination of the physical parameters of loops, such as the magnetic field, density and temperature that are difficult to measure with other methods. We present the results of recent 3D MHD models of waves in active region loops motivated by recent observations. Waves in dipole and constant- $\alpha$ force-free field with gravitationally stratified density were studied by Ofman and Thompson (2002). High density coronal loop was introduced in the above configuration by McLaughlin and Ofman (2008). Waves in more realistic active region initialized with potential magnetic field extrapolated from observed photospheric field were studied by Ofman (2007). We show how the effects of active region morphology, twist, and flow influence the oscillation of the loops. 

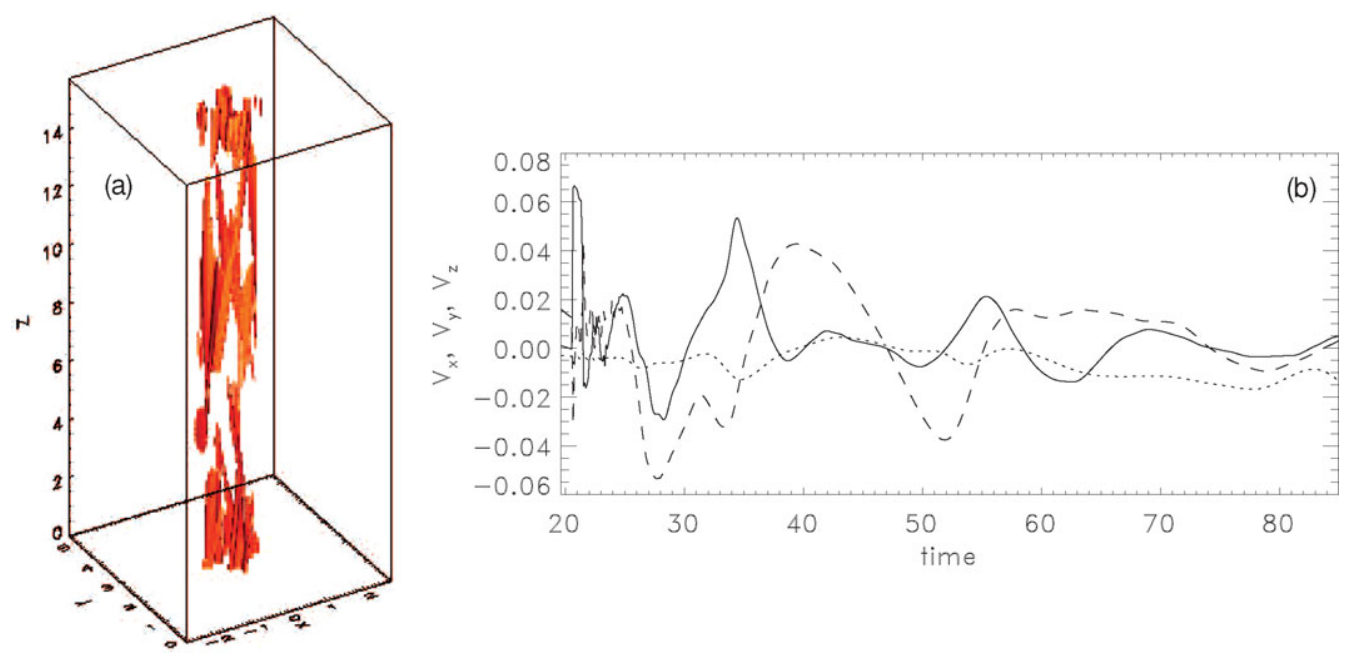

Figure 1. The isosurface of the current density $j^{2}$ in the twisted loop, formed as a result of the twist and the impact of the wave.

\section{MHD model}

We describe solar plasma with the normalized three-dimensional nonlinear resistive MHD equations with gravity included for the dipole case. For simplicity we study the isothermal case. Detailed description of the code can be found Ofman \& Thompson (2002).

\subsection{Twisted Loops}

The four-threaded loop is initialized with

$$
\rho_{0}(x, y, z)=\rho_{\min }+\left(\rho_{\max }-\rho_{\min }\right) \sum_{i=1}^{4} e^{-\left\{\left[\left(x-x_{i}\right)^{2}+\left(y-y_{i}\right)^{2}\right] / r_{0}^{2}\right\}^{2}},
$$

where $\rho_{\min }=0.2$ is the minimal normalized density outside the loops, $\rho_{\max }=1$ is the maximal normalized density of the loops, $r_{0}=0.25$ is the radius of the loops, and $\left(x_{i}, y_{i}\right)$, where $i=1,2,3,4$ are the locations of the axes of the four threads. The twist is introduced by applying circular flow in the $x-y$ plane at $z=0$ for quarter rotation. Line-tied boundary conditions are used at the top and the bottom of the simulation box, and open boundary conditions on the sides. We launch a velocity pulse that impacts the four-threaded loops and initiates the oscillations.

\subsection{Loops in Dipole Field}

As the initial configuration we take a potential dipole magnetic field and assume a gravitationally stratified equilibrium density (Ofman \& Thompson 2002). Following McLaughlin \& Ofman (2008) we include a denser loop in our system, however we construct it in such a way that it follows fieldlines and has a smooth density profile:

$$
\varrho_{\mathrm{i}}=\varrho_{\mathrm{e}} d \exp \left[-\left(\left[\left(y-y_{0}\right)^{2}+\left(z-z_{0}\right)^{2}\right] / w\right)^{p}\right] .
$$

At the photospheric boundary we keep all the variables fixed while open boundary conditions are implemented for all the variables at the other five planes. Note, that due to the rapid drop in the magnetic field density, the plasma $\beta$ increases with height, and it is about 2 at the apex of the loop. 

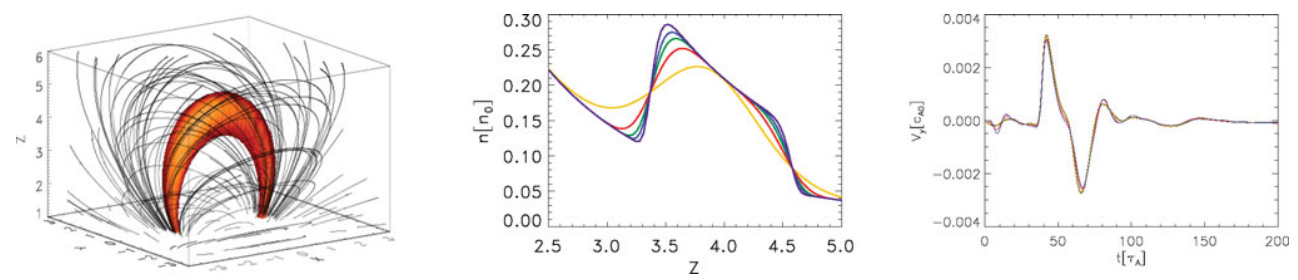

Figure 2. Left panel: Initial 3D magnetic field configuration. Middle panel: Different density profiles (along $\mathrm{x}=\mathrm{y}=0$ cut) of the loop: $\mathrm{p}=6$ (violet line), $\mathrm{p}=4$ (blue line), $\mathrm{p}=3$ (green line), $\mathrm{p}=2$ (red line) and $\mathrm{p}=1$ (yellow line). Right panel: Time signatures of transversal component of velocity. Colors correspond to middle panel notation.

\section{Numerical Results}

\subsection{Twisted Loops}

We find that the twisted loop exhibits transverse oscillations due to the impact of the velocity pulse. The oscillations are more complex than a kink mode in a straight cylinder, and all 3 components of the velocity exhibit damped wave motions. Filamented currents form in the loop due to the combined effects of the twist and the waves (see Figure 1a). The damping rate of the twisted loop is faster, and the phase speed is larger than the kink speed of the parallel-threaded loop case (see Figure 1b).

\subsection{Loops in Dipole Field}

We start our studies by perturbing the loop with a velocity pulse from the side boundary plane. We vary the steepness parameter of the density ratio: $p=1,2,3,4,6$. predicted for a straight cylinder that resonance layer width should affect the damping time of the oscillation through the resonant layer width and the density ratio between the loop and surrounding corona.

From the middle panel of Fig. 2 we see that the width of inhomogeneous layer changes by the factor of 5 between steepness parameters $p=1$ and $p=6$ for a fixed density ratio. According to Ruderman \& Roberts' (2002) theory we should expect the difference in normalized damping times up to $20 \%$. However, we find that resonant absorption does not affect the damping in curved loops (right panel of Fig. 2), because by the time it starts to act the oscillations are damped due to wave leakage enhanced by the large $\beta$ near loop apex, and the loop curvature.

Next we model the observation of slow standing waves reported by Wang et al. (2003a,b). We excite the slow standing wave by a velocity pulse (duration of the pulse $\ll$ waveperiod) from the bottom boundary plane centered in one of loop's footpoints. As the effect of such a pulse launched in the footpoint we observe the initial brightening (left panel of Fig. 3) which is consistent with slow wave oscillation observations with SXT (Fig. 8 from Wang et al. 2003b). This kind of excitation produces two pulses launched in both footpoints of the loop simultaneously due to the fast wave coupling (Selwa et al. 2007). The time signatures of mass density and velocity at the apex shown in right panel of Fig. 3) match the observational ones (quarter wave-period shift and initial antiphase stage; compare with Fig. 3 from Wang et al. 2003a). However, the damping rate is more rapid in our model due to the large value of $\beta$ and the curvature which lead to increased leakage of the slow wave. 

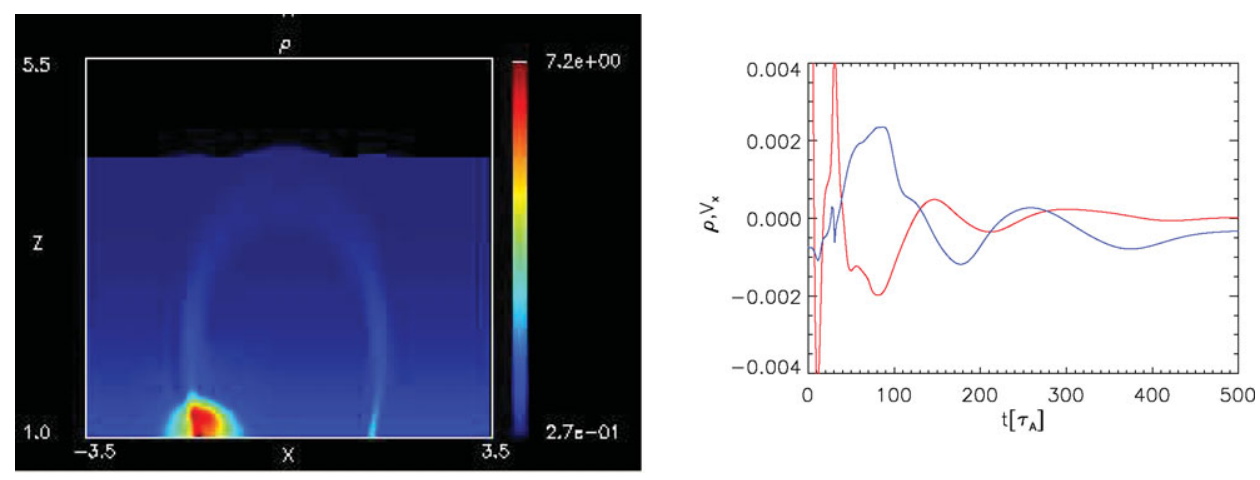

Figure 3. Left: Initial brightening due to the pulse in one of the loop's footpoint. Right: Time signatures of mass density and velocity at the apex.

\section{Conclusions}

Motivated by recent observations we model the transverse oscillations of a twisted four-threaded loop. We find that the oscillations of the loop are more complex than the kink mode, with larger phase speed and damping rate than in parallel-thread loop.

For the loop in the dipole field we observe no difference in damping rates due to steepness of density profile across the loop since the dominant damping mechanism is leakage of the fast mode wave due to large plasma $\beta$ in the loop. We find that for a curved loop footpoint excitation is an efficient mechanism of triggering the slow standing mode: the main observational features (excitation within 1 wave-period and initial footpoint brightening) are reproduced. The results of our study are useful for the development of coronal seismology methods.

\section{Acknowledgement}

The authors thank support by NASA grants NNG06GI55G and NNX08AV88G.

\section{References}

Aschwanden, M. J., Fletcher, L., Schrijver, C. J., \& Alexander, D. 1999, ApJ, 520, 880

McLaughlin, J. A. \& Ofman, L. 2008, ApJ, 682, 1338

Nakariakov, V. M., Ofman, L., DeLuca, E., Roberts, B., \& Davila, J. M. 1999, Science, 285, 862

Ofman, L. 2007, ApJ, 655, 1134

Ofman, L. \& Thompson, B. J. 2002, ApJ, 574, 440

Ofman, L. \& Wang, T. J. 2008, Astron. Astrophys., 482, L9

Ruderman, M. S. \& Roberts, B. 2002, ApJ, 577, 475

Selwa, M., Ofman, L., \& Murawski, K. 2007, ApJL, 668, L83

Verwichte, E., Nakariakov, V. M., Ofman, L., \& Deluca, E. E. 2004, Sol. Phys., 223, 77

Wang, T. J., Solanki, S. K., Curdt, W., Innes, D. E., Dammasch, I. E., \& Kliem, B. 2003a, Astron. Astrophys., 406, 1105

Wang, T. J., Solanki, S. K., Innes, D. E., Curdt, W., \& Marsch, E. 2003b, Astron. Astrophys., 402, L17 